\title{
Macht, Markt, Mitverantwortung
}

\author{
Wie können Unternehmen und deren Verbände Einfluß auf den umweltpoliti- \\ schen Willensbildungsprozeß nehmen? Dieser Frage wird unter anderen die \\ VöW-Jahrestagung Anfang Dezember nachgehen. Die grundsätzlichen Perspek- \\ tiven unfernehmerischer politischer Mitverantwortung, die Rolle von Unterneh- \\ mensverbänden und die Bedeutung kooperativer Lösungsansätze \\ sind Schwerpunkte der Diskussion.
}

$\mathrm{D}_{\mathrm{s}}$ in vollem Gange, so scheint es zumindest: Sowohl auf der Agenda internationaler, nationaler und kommunaler Politik als auch als neuer Orientierungspunkt ökologischer Unternehmensführung. Von Rio-Nachfolgeprozessen über nationale Umweltpläne und EnqueteKommissionen bis zu zahlreichen Agenda 21Prozessen auf lokaler Ebene reicht das Spektrum. Und auch Unternehmen und Wirtschaftsverbände haben sich „Nachhaltigkeit" auf die Fahnen geschrieben. Von den Mitgliedern des World Business Concil for Sustainable Development über die Unterzeichner der ICC-Charta für Nachhaltige Entwicklung, das Responsible Care-Programm der Chemieindustrie bis zu den Initiativen zahlreicher Einzelunternehmen wird Nachhaltigkeit heute in der Wirtschaft gefo(e)rdert. In einer Anhörung der Enquete-Kommission „Schutz des Menschen und der Umwelt" zu Nachhaltigkeitskonzepten in der deutschen Wirtschaft wurde vor kurzem deutlich, welche breite Resonanz das ,Unternehmen Nachhaltigkeit" auch in den Managmentetagen gefunden hat (1).

\section{- "Unternehmen Nachhaltigkeit" - eine Zwischenperspektive}

Doch trotz dieses breiten Engagements bleiben die ökologischen Erfolge bisher aus: Grundsätzlichen Managementregeln einer nachhaltigen Entwicklung werden kaum eingehalten, wie die meisten ökologischen Belastungsindikatoren zeigen. Die neuesten ökologischen Daten der volkswirtschaftlichen Gesamtrechnung für Deutschland weisen zwar eine Entkopplung der ökologischen Belastungen vom Bruttoinlandsprodukt auf, jedoch haben sich die meisten Indikatoren absolut lediglich stabilisiert und sind von den häufig geforderten Reduktionen um einen Faktor vier oder sogar Faktor zehn weit entfernt. Und selbst die Einhaltung der von der Bundesregierung zugesagten C02-Reduktion um 25 Prozent bis zum Jahre 2005 scheint trotz Selbstverpflichtungen der deutschen Industrie - kaum einhaltbar (2). Die Gründe für diese ernüchternde ökologische Bilanz sind vielfältig. Der umweltpolitische Gestaltungswille der politischen Entscheidungsträger scheint durch den sozialen und wirtschaftichen Pol des Nachhaltigkeitsdreiecks von „Ökologie-ÖkonomieSoziales" bedroht zu sein: Massenarbeitslosigkeit und Wettbewerbsfähigkeit des Standortes Deutschland stehen oben auf der politischen Agenda. Und auch Unternehmen sehen ihre zentralen Herausforderungen eher im globalen Wettbewerb als in der Lösung ökologischer Probleme - aller Nachhaltigkeitsbekundungen zum Trotz.

Gerade in einer solchen Zeit erscheint es notwendig, sich mit Offenheit Pfaden für-das „Unternehmen Nachhaltigkeit“ zuzuwenden, die das fragile Gleichgewicht aus ökologischen, ökonomischen und sozialen Anforderungen berïcksichtigen. Solche Pfade können weder durch politische Entscheidungen noch durch das Umweltmanagement von Unternehmen alleine geschaffen werden. Zur Überwindung seiner „pragmatischen Ohnmacht“(3) ist der Umweltstaat vielmehr darauf angewiesen, daß Unternehmen ihre ökologischen Handlungs- und Gestaltungsmöglichkeiten konstruktiv in die gemeinsame Politikfindung einbringen und damit helfen, einige der Barrieren niederzureißen, die dem Unternehmen Nachhaltigkeit heute noch entgegenstehen. Die diesjährige vöW-Jahrestagung geht daher den Einflußpotentialen und Handlungsmöglichkeiten der Unternehmen und Unternehmensverbänden für eine politische Mitverantwortung nach.

\section{Grundsätzliche Perspektiven}

Eine politische Sicht der Unternehmung hat sich bisher weder in der betriebswirtschaftlichen Theorie noch in den Managementverständissen der Praktiker in Unternehmen durchsetzen können. Einzelne theoretische Versuche wie die Arbeiten von Peter Ulrich (4) konnten sich kaum gegen den Strom der Literatur mit management- und sozialtechnischem Verfiigungswissen behaupten. Und in den Führungsetagen von Unternehmen wird hauptsächlich die Verantwortung für den Shareholder-Value betont. Politische Mitverantwortung wird dagegen kaum gesehen - oder sogar explizit abgestritten - wie im Fall Shell und Nigeria, in dem das Unternehmen sich gegen jede Einflußnahme auf die politischen Verhältnisse in dem afrikanischen Land verwehrt.

Dabei gibt es kein unpolitisches Handeln von Unternehmen. Unternehmen und Wirtschaftsverbände sind ein Teil unserer Gesellschaft - ihr Handeln prägt daher zwangsläufig Politik und Öffentlichkeit mit. Da das „Unternehmen Nachhaltigkeit" auf die enge Koordination von Wirtschaft und Politik angewiesen ist, scheint es fruchtbar, sich diesem Zusammenspiel intensiver $\mathrm{zu}$ widmen und Handlungsperspektiven für Unternehmen aufzuzeigen. Diese Notwendigkeit wird durchaus auch von einzelnen Unternehmen und Verbänden gesehen: So veranstalten z.B. VCI und IG Chemie derzeit eine umfangreiche Workshopreihe mit zahlreichen externen Experten, um die entsprechenden Handlungsspielräume von Unternehmen systematisch zu evaluieren. Allgemeine Fragen, die es zu diskutieren gilt sind unter anderen:

- Was sind die Mechanismen, mit denen Unternehmen in marktliche und politische Prozesse eingreifen?

- Wie groß sind die Handlungsspielräume der Unternehmen, um über solche Mechanismen „ordnungspolitische Mitverantwortung“ auszuüben?

- Gibt es bestimmte Unternehmen oder Handlungsformen, denen in diesem Prozeß eine besondere Bedeutung zukommt?

Diese Fragen bewegen sich im Schnittbereich von Betriebswirtschaftslehre, Volkswirtschaftslehre, Politologie und Soziologie und fordern auf zu einem interdisziplinären Brückenschlag. Auf der Jahrestagung werden daher einerseits unterschiedliche theoretische Zugänge betrachtet (Pfriem / Hockerts, Christ), andererseits die Erfahrungen aus ein- 
zelnen Unternehmen (Knoblauch, Hewlett Packard) und von Praktikern des politischen Prozesses (Hustedt, Bündnis90/Die Grünen) vorgestellt.

\section{Rolle der Verbände}

Von Selbstverpflichtungen über öffentliche Stellungnahmen bis zur Teilnahme an EnqueteKommissionen: Es sind selten Einzelunternehmen, die im politischen Geschäft auftreten, sondern in der Regel Verbände. Zu den klassischen Unternehmensverbänden sind in den letzten Jahren ökologische Unternehmensverbände wie B.A.U.M., future oder UnternehmensGrün hinzugekommen.

Welchen Einfluß nehmen Verbände heute auf das „Unternehmen Nachhaltigkeit? Was bestimmt die Höhe ihrer „Macht"? Auf welche Weise nehmen sie auf politische und öffentliche Prozesse Einfluß? Wie ist die „Macht“ der neu entstandenen Unternehmerverbände zu beurteilen? Haben sie „Machtgleichgewichte“ im politischen Geschäft verschoben? Welche Rolle können und sollen Verbände in der Gestaltung des „Unternehmens Nachhaltigkeit“ spielen? Auf der VÖW-Jahrestagung kommen zu diesem Themenkomplex sowohl die Vertreter klassischer großer Industrieverbände (BDI, Becker / VCI, Druckrey) als auch von grïnen Unternehmensverbänden (Rock, UnternehmenGrün) sowie von „ergrünenden“ Verbänden (Wenz, BJU) zu Wort.

\section{Ökologische Markfgestaltung}

Die politische Mitgestaltung durch Unternehmen geschieht heute zunehmend kooperativ. Dabei bezieht sich diese Kooperation nicht nur auf die Zusammenarbeit von Unternehmen innerhalb von Verbänden: Von vertikalen Kooperationen entlang der Stoff- und Wertschöpfungsketten über Branchenvereinbarungen und Regionalkooperationen bis zur Zusammenarbeit von Umweltschutzverbänden und Unternehmen reicht das Spektrum kooperativer ökologischer Mitgestaltung von Unternehmen.

Welchen Beitrag leisten diese Kooperationen zu einer Ökologisierung unseres Wirtschaftens? Welche Kooperationsformen erweisen sich diesbezïglich als besonders effektiv? Wie sollte sich korporatistische Umweltgestaltung in Zukunft weiterentwickeln?

Aufbauend auf den ersten theoretischen Arbeiten zu ökologischen Unternehmenskooperationen (5) sowie zahlreichen praktischen Erfahrungen, werden diese Fragen auf der vöW-Jahrestagung diskutiert. Dabei stehen sowohl übergeordnete Aspekte „neuer Koalitionen“ als auch spezifische Ausprägungen von Kooperationen (insbesondere Regionalkooperationen) auf der Tagesordnung. Die konzeptionellen Aspekte werden durch Beispiele abgerundet: Die aktuellen Wirtschaftskooperationen von Greenpeace kommen dabei genauso zur Sprache wie aktuelle Kooperationen im regionalen Kontext.

Durch die Mischung theoretischer und praktischer Beiträge möchte die Tagung dazu beitragen, das Handeln von Unternehmen in Spannungsfeld von Macht, Markt und politischer Mitverantwortung einerseits besser zu verstehen und andererseits konkrete Ansätze zur Förderung des ,Unternehmens Nachhaltigkeit“ zu entwickeln. Die wichtigsten Beiträge der Tagung sollen in einem Buch dokumentiert werden, um die in Berlin initiierte Diskussion auch anderen Interessierten zugänglich zu machen und die Diskussion über die Veranstaltung hinaus wachzuhalten.

Die Autoren
Uwe Schneidewind ist wissenschafflicher Mitarbeiter am
Institut für Wirtschaft und Ökologie (IWÖ), St. Gallen.
Heinrich Strößenreuther ist Vorstand des VÖW.
Kontakte: Schneidewind, IWÖ-HSG, (H-St. Gallen,
Tel. (++41) (71) 224-2584;
Strößenreuther, Bonn, Tel. (0228) 16-23113

\section{Anmerkungen}

1) Enquete-Kommission: „Nachhaltigkeitskonzepte in der Wirtschaft". Fragen- und Sachverständigenkatalog sowie Stellungnahmen für die öffentliche Anhörung „Nachhaltigkeitskonzepte in der Wirtschaft" der Enquete-Kommission "Schutz des Menschen und der Umwelt" am 29. und 30. April 1996. Kommissionsdrucksache 13/1, 13/1 a-g. Deutscher Bundestag. Bonn 1996

- H. Strößenreuther: Corporate-Driven Political Initiatives for Sustainable Development. Tagungsbeitrag zur Fifth International Conference of The Greening of Industry Network "Global Restructuring" vom 24.-27.11.1996 in Heidelberg. 2) H. Bergmann, L. Brockmann, K. Rennings: Möglichkeiten und Grenzen von freiwilligen Umweltschutzmaßnahmen der Wirtschaft unter ordnungspolitischen Aspekten. ZEWGutachten. Mannheim 1996.

3) T. Kuhn: Unternehmerische Verantwortung in der ökologischen Krise als "Ethik der gestaltbaren Zahlen". Unternehmensethische Leitlinien für umwelt- und erfolgsbewuftes Management. Bern U.a. 1993, S. 161.

4) P. Ulrich: Die Großunternehmung als quasi-öffentliche Institution. Eine politische Theorie der Unternehmung. Stuttgart 1977

- P. Ulrich: Integrative Wirtschafts- und Unternehmensethik ein Rahmenkonzept, in: Forum für Philosophie (Hrsg.): Markt und Moral: die Diskussion um die Unternehmensethik. Bern u.a. 1994, S. 75-107.

5) A. Aulinger: (Ko)-Operation Ökologie. Kooperation im Rahmen ökologischer Unternehmenspolitik. Marburg 1996 - M. Brockhaus: Gesellschaftsorientierte Kooperationen. Wiesbaden 1996

- U. Schneidewind: Ökologisch orientierte Kooperationen aus betriebswirtschaftlicher Sicht, in: uwf 3. Jg., H. 4. Dezember 1995, S. 16-21

\section{Ökologie und Partizipation}

\section{Zwei Ziele bestimmen seit den 80 er Jahren die Diskussion einer modernen, innovativen Unfernehmensführung: Die betriebliche Ökologisierung sowie die Demokratisierung der Entscheidungs- und Handlungsprozesse. Wie können Ökologie und innerbetriebliche, regionale sowie politische Partizipation sinnvoll zusammenwirken?}

$\mathrm{B}$ estehende Erfahrungen mit der Umsetzung einer ökologisch partizipativ orientierten Wirtschaftsweise zusammenzuführen, ist das Ziel des Projektes „Ökologie und Partizipation“. Aus Deutschland, Österreich, England, Irland, Italien und Schweden wurden bereits in Form von Fallstudien die Erfahrungen kleiner und mittlerer Betriebe dazu gesammelt und die jeweiligen Grenzen unter den bestehenden Rahmenbedinungen herausgearbeitet. Dazu wurden Interviews in einzelnen Betrieben - in Deutschland 20; England 15; Irland, Österreich und Schweden fün - durchgefïhrt. Die untersuchten Betriebe arbeiten in den Bereichen Lebensmittel, Bau, Energie und Verkehr. So wurden beispielsweise im Bereich Lebensmittel unter anderem die Verbraucher- und Erzeugergenossenschaft für naturgemäßes Leben, Tagwerk und die Firma Bruno Fischer; im Naturkosthandel die Firmen Sonnenblume und Cibaria 
(c) 20I0 Authors; licensee IÖW and oekom verlag. This is an article distributed under the terms of the Creative Commons Attribution Non-Commercial No Derivates License (http://creativecommons.org/licenses/by-nc-nd/3.o/), which permits unrestricted use, distribution, and reproduction in any medium, provided the original work is properly cited. 